\title{
纯磑化铯(CsI)晶体的发光与光衰减特性研究
}

\author{
任国浩 ${ }^{1}$, 宋朝晖 ${ }^{2}$, 张子川 ${ }^{2}$, 张 㑆 ${ }^{2}$, 杨 帆 $^{1}$, 李焕英 ${ }^{1}$, 陈晓峰 ${ }^{1}$ \\ (1.中国科学院 上海硅酸盐研究所, 上海 201800; 2. 西北核技术研究所，西安 710024)
}

摘 要: 纯碘化铯晶体是一种具有快衰减闪秌特性的新型辐射探测材料, 在伽马射线、中子和其它辐射探测技术中 有重要的应用前景。本研究以 Bridgman 法所生长的纯碘化铯为对象, 分别研究了该晶体的透射光谱以及在紫外 光、连续 X 射线、脉冲 $\mathrm{X}$ 射线和宇宙射线激发下的发射光谱、时间响应特性和沿晶体生长方向不同部位的光输出 分布特点。实验测得晶体的吸收边为 $240 \mathrm{~nm}$, 激发和发射波长分别位于 $241 \mathrm{~nm}$ 和 $318 \mathrm{~nm}$, 发光衰减时间分别为 2 3 $\mathrm{ns}$ 和 18 25 ns。以尺寸为 $30 \mathrm{~mm} \times 30 \mathrm{~mm} \times 200 \mathrm{~mm}$ 的晶体籽晶端和尾端与 PMT 耦合所测得的光输出分别是 143 p.e/MeV 和 127 p.e./MeV, 尽管晶体两端的光输出存在 $12.6 \%$ 的差异, 但没有观察到衰减时间长于 $100 \mathrm{~ns}$ 的慢 分量。这些性能进一步证明纯碘化铯晶体具有作为快闪炼体的优势。

关 键 词: 纯碘化铯; 晶体; 透光性; 发光; 衰减时间; 光输出

中图分类号: O734 文献标识码: A

\section{Luminescence and Decay Time Properties of Pure CsI Crystals}

\author{
REN Guo-Hao ${ }^{1}$, SONG Zhao-Hui ${ }^{2}$, ZHANG Zi-Chuan ${ }^{2}$, ZHANG Kan², YANG Fan ${ }^{1}$, LI Huan-Ying ${ }^{1}$, \\ CHEN Xiao-Feng ${ }^{1}$
}

(1. Shanghai Institute of Ceramics, Chinese Academy of Sciences, Shanghai 201800, China; 2. Northwestern Institute of Nuclear Technology, Xi'an 710024, China)

\begin{abstract}
Pure CsI crystal is a kind of fast scintillators which can be used to detect $\gamma$-rays, neutrons as well as other radiation. Optical transmission and luminescence properties of pure CsI crystal grown with Vertical Bridgman method were measured under the excitation of UV, continuous X-ray, pulse X-ray as well as cosmic rays at room temperature. Cut-off edge of the grown CsI crystal reaches $240 \mathrm{~nm}$, which is the shortest among the literature reported previously. Excitation and emission wavelength are $241 \mathrm{~nm}$ and $318 \mathrm{~nm}$, respectively. The decay times of the fast intrinsic luminescence can be fitted into two components, $2 \sim 3 \mathrm{~nm}$ and 18 $25 \mathrm{~nm}$ under the excitation of X-ray or cosmic rays. The light outputs measured from the crystal with size of $30 \mathrm{~mm} \times 30 \mathrm{~mm} \times 200 \mathrm{~mm}$ are $143 \mathrm{p}$.e/MeV and 127 p.e./MeV as its seed end and tail end were coupled with PMT, respectively. Even though the non-uniformity (12.6\%) exists along the growth direction of the crystal, no significant slow component longer than $100 \mathrm{~ns}$ are observed. All of these properties prove that the pure CsI crystal is a promising fast scintillator.
\end{abstract}

Key words: pure CsI; crystal; transmittance; luminescence; decay time; light output

具有快衰减时间特性的闪炼体一直是高能物理 实验、核物理和正电子断层扫描仪等技术所追求的 辐射探测材料, 它有利于高计数率和纳秒、亚纳秒量 级的时间分辨 ${ }^{[1]}$ 。氟化钡 $(0.6 \mathrm{~ns})^{[2]}, \gamma-\operatorname{CuI}(0.13 \mathrm{~ns})^{[3]}$ 、
$\mathrm{ZnO}: \mathrm{Ga}(0.7 \mathrm{~ns})$ 和纯碘化铯是公认的快闪炼体，但 $\mathrm{BaF}_{2}$ 的发光波长位于 $220 \mathrm{~nm}$ 的紫外区, 这给光探 测带来了困难。 $\gamma-\mathrm{CuI}$ 半导体生长困难, 至今只能生 长厘米量级的晶体, 离实际应用还有较大的差距; 
$\mathrm{ZnO}: \mathrm{Ga}$ 的慢分量控制非常困难。相比较而言, 纯 CsI 晶体的衰减时间 $<10 \mathrm{~ns}$, 而且它是立方晶系, 对称程度高, 方便生长出较大尺寸的块体单晶。所 以日本 KEK 已经选用纯 CsI 晶体对其 Supper Belle 电磁量能器的端帽探测器进行更新 ${ }^{[4]}$, 美国费米实 验室在经过反复论证之后, 也决定在其即将建造的 Mu2e 工程中采用纯碘化铯晶体作为电磁量能器的 核心探测材料 ${ }^{[5]}$ 。

日本学者 KUBOTA 等 ${ }^{[6]}$ 最早报道了纯 CsI 晶体 的快衰减特性。为了探索纯 CsI 晶体在高能物理领 域应用的可能性, 美国加州理工学院对乌克兰单晶 研究所提供的纯 CsI 晶体做了进一步研究, 发现该 晶体存在两个发光分量, 其主要发光波长为 $310 \mathrm{~nm}$, 并存在一个波长为 $450 \mathrm{~nm}$ 的慢分量, 快/慢分量比 为 $30 / 70^{[7]}$, 抗辐照硬度是 $\operatorname{CsI}(\mathrm{Tl})$ 的 10 倍 ${ }^{[8]}$ 。特别 是, 相对于 $\mathrm{BaF}_{2}$ 晶体, $\mathrm{CsI}$ 较短的辐射长度 $(1.86 \mathrm{~cm})$ 和较低的密度 $\left(4.51 \mathrm{~g} / \mathrm{cm}^{3}\right)$ 可使电磁量能器的体积和 重量分别缩小 $15 \%$ 和 $22 \%{ }^{[7]}$ 。但根据 Amsler 的研究, 纯 CsI 晶体的光产额在室温下只有 $\mathrm{CsI}(\mathrm{Tl})$ 晶体的 $5 \%$ 左右, 并且会随着温度的升高而下降 ${ }^{[9]}$ 。较低的 光产额和较低的快/慢成分比对实际应用是不利的。 因此, 能否制备出具有较高光输出和较高快/慢分量 比的纯 CsI 便成为该晶体能否获得实际应用的关 键。另外受原料质量和晶体制备工艺的限制, 不同 研究者报道的纯 CsI 晶体的闪炼性能并不完全相同, 特别是晶体光衰减时间存在很大的差异。为此, 本 工作针对我们新近生长的纯 CsI 晶体进行了发光和 光衰减性能研究。

\section{1 实验方法}

以纯度为 99.999\%的 CsI 多晶粉末为原料, 先在 真空下烘干原料中的吸附水, 然后装入石英玻璃坩

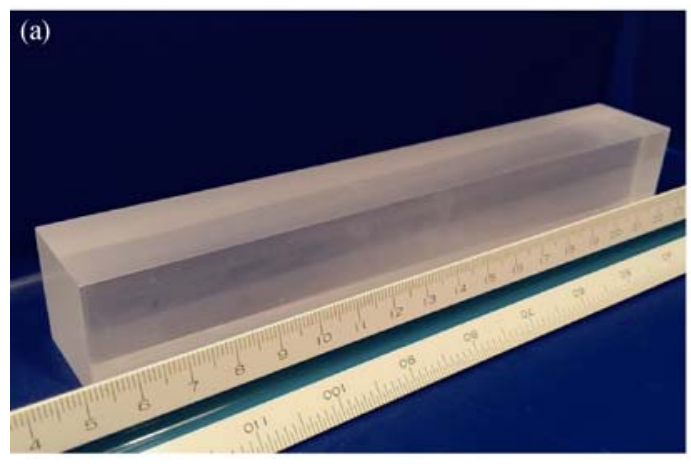

埚中再对坩埚抽真空并封口。已知 CsI 晶体为立方 原始格子, 空间群为 $\mathrm{Pm} 3 \mathrm{~m}$, 晶胞参数 $a=0.45679 \mathrm{~nm}$, 无解理，属于各向同性的晶体，因此实验所使用的 籽晶没有选择特定的结晶学方向。采用垂直 Bridgman 方法进行晶体生长, 生长出的毛坏晶体体 积为 $\phi 50 \mathrm{~mm} \times 350 \mathrm{~mm}$, 晶体经过切割、研磨和抛光 后制作成规定形状的样品。采用 Shimadzu UV-2501PC 分光光度仪测试晶体的透光特性, 采用 Perkin-Elmer LS 50B 紫外苂光光谱仪测试晶体的 激发和发射光谱, 以连续 $\mathrm{X}$ 射线为激发源的单色仪 测试晶体的发射光谱, 以脉冲 $X$ 射线和宇宙射线为 激发源分别测试了晶体的光衰减曲线, 并对这些曲 线进行拟合。以 ${ }^{22} \mathrm{Na}$ 为激发源和 HAMAMATSU 的 R2059 光电倍增管(PMT)为光探测器, 分别以晶体 籽晶端和尾端与 PMT 耦合测试了晶体的光输出。

\section{2 结果与讨论}

\section{1 透光特性}

将尺寸为 $27 \mathrm{~mm} \times 35 \mathrm{~mm} \times 200 \mathrm{~mm}$ 的纯 CsI 晶 体六面抛光后(图 1(a)) 分别沿长轴和垂直于长轴方 向测试晶体的透射光谱(图 1b), 从图中发现，当沿 垂直长轴方向测试时, 透光率从可见光区的 $70 \%$ 以 上陡然下降到紫外区的零, 截止吸收边落在 $240 \mathrm{~nm}$ 处(图 1(b)), 这与文献[9-10]报道的吸收边(248 nm 和 $250 \mathrm{~nm}$ )相比更加靠近紫外区，非常逼近纯 CsI 的 理论吸收边(CsI 的禁带宽度 $E_{\mathrm{g}}=6.1 \mathrm{eV}$ ), 说明本实 验生长的晶体比文献报道的纯 CsI 晶体的质量有了 明显提高。而当沿平行于长轴方向测试时, 吸收边会 出现 3 5 nm 的红移, 而且透过率也明显降低(图 1(b)), 这是由于晶体自身对光的吸收和散射作用随光程的 延长而增强所致。同时，经仔细观察还会发现，无 论是纵向还是横向透射光谱, 它们在 $300 \mathrm{~nm}$ 附近都

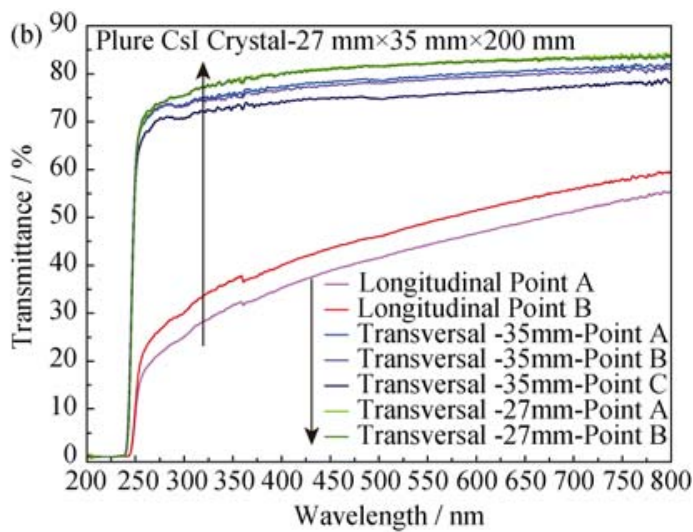

图 1 纯 CsI 晶体的照片(a)及其轴向与横向透射光谱图(b)

Fig. 1 Photo (a) and longitudinal and transversal transmittance (b) of pure CsI crystal 
会有一个微弱的吸收谷, 通过与掺铊碘化铯晶体对 比 $^{[11]}$, 该吸收谷应该是由于晶体中的痕量铊污染所 致(因掺铊碘化铯晶体也是本实验室的一个主要研 究对象, 存在共用坩埚和容器而造成交叉污染的可 能性)。而 $360 \mathrm{~nm}$ 的突变则是由于两种光源切换所 产生台阶。

\section{2 发光特性}

用紫外菼光光谱仪测试了纯 CsI 晶体的激发和 发射光谱, 结果显示(图 2), 纯 CsI 晶体的激发波长 位于 $241 \mathrm{~nm}$, 发射波长位于 $318 \mathrm{~nm}$ 。这个发射波长 与 Schotanus 报道的 $315 \mathrm{~nm}^{[8]}$, Amsler 和 Woody 报道的 $310 \mathrm{~nm}$ 发射峰比较接近 ${ }^{[12-13]}$ 。由于纯 CsI 晶 体的发射波长位于紫外区, 给光探测带来一定的困 难, 实际工作中需采用对紫外光敏感的光探测器, 或者在晶体表面涂上一层能够将紫外光移至可见光 的苂光染料(波长位移膜), 以获得好的探测效果。 而文献[10]中则没有测到这一紫外发射峰，只在 $450 \mathrm{~nm}$ 探测到纯 CsI 晶体的非本征发射。图 2 还显 示, 除了 $318 \mathrm{~nm}$ 这个主发光峰之外, 该晶体在 425 $700 \mathrm{~nm}$ 波段存在微弱的发射带, 该发光带属于晶体 中存在的少量慢发光成分。

以铇靶在 $55 \mathrm{kV}$ 电压下所产生的 $\mathrm{X}$ 射线为激发 源, 测试了几个纯碘化铯晶体的发射光谱(图 3), 尽 管所有样品都是在相同条件下测试的, 但不同样品 的发射强度差别较大, 主发射峰的波长存在微小的 差异, 变化范围介于 312 320 nm 之间, 这个波长与 用紫外光激发所测得的发射波长(图 2, $318 \mathrm{~nm}$ )基本 一致。同样，该 X 射线激发发射谱在 400 575 nm 之间也存在强度较弱的发光带, 属于慢发光成分, 除 CsI-3 样品之外, 多数样品的快/慢分量比都大于

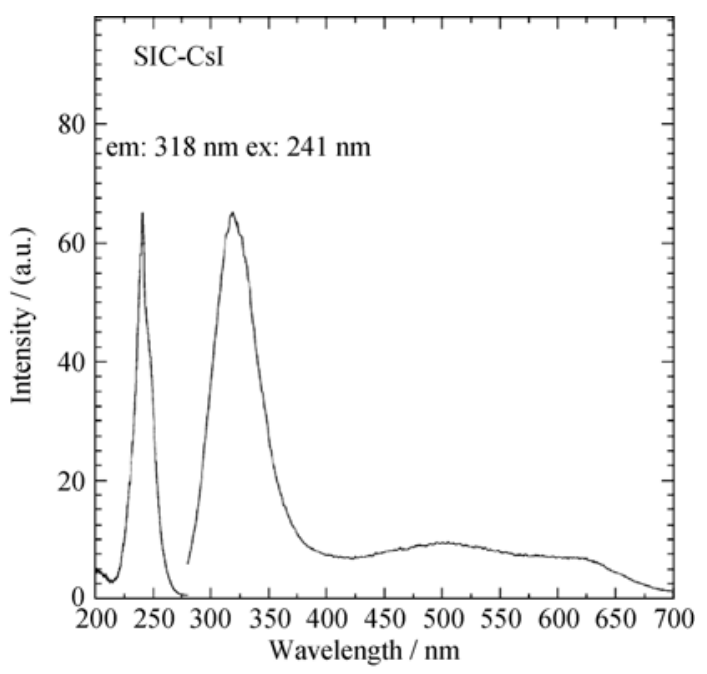

图 2 纯 CsI 晶体的紫外激发发射光谱图

Fig. 2 Photoluminescence spectra of pure CsI crystal

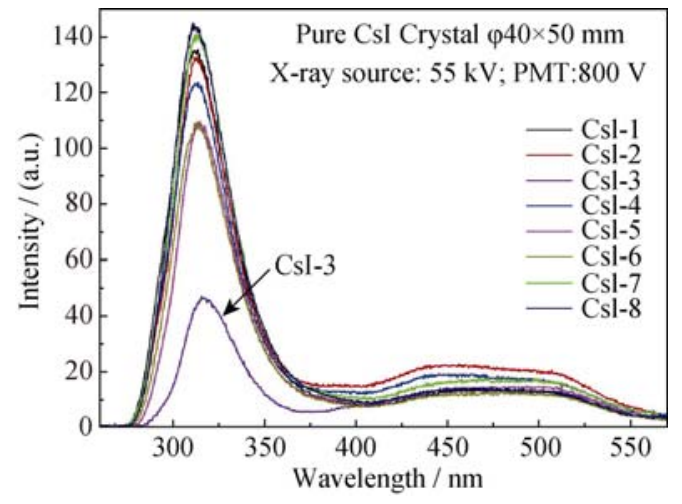

图 3 纯 CsI 晶体的 X 射线激发发射光谱图

Fig. 3 X-ray excited luminescence (XEL) spectra of pure CsI crystals

80/20，此值远大于文献[9]中报道的 30/70, 说明与 慢分量有关的缺陷在本实验测试的样品中得到了有 效抑制。

对于纯 CsI 晶体在 312 320 nm 之间的主发光峰, 即所谓的本征快发光, 一般认为它起源于晶体的本 征发光。但实际上，这个快分量并非单一发光组分， 而是由两个组分所构成, 根据低温下的测试结果 ${ }^{[8]}$, 这两个组分的发光波长分别为 $305 \mathrm{~nm}$ 和 $340 \mathrm{~nm}$, 而且它们的强度随温度和 X 射线管电压的变化呈现 不同的变化趋势, 其成因源于晶体中的本征缺陷, 分别归属于 $\left(\mathrm{V}_{\mathrm{k}}+\mathrm{e}\right)$ 和 $(\mathrm{H}+\mathrm{F})$ 型自陷激子发光。而波长 位于 400 700 nm 之间的发光带, 即所谓的慢成分, 其成因则存在比较大的争议。多数研究者认为慢分 量起源于碘空位所引起的晶格缺陷 ${ }^{[9,14]}$, 或者是占 据碘空位的氧杂质缺陷 ${ }^{[10]}$ 。Gektin 的掺杂试验表明, $\mathrm{Na} 、$ Ca 掺杂的 CsI 都会在 $420 \mathrm{~nm}$ 处产生发光峰; Sb、 Bi 掺杂 CsI 除了产生 $420 \mathrm{~nm}$ 发射峰, 还会在 $550 \mathrm{~nm}$ 处产生发光峰; $\mathrm{Pb}$ 掺杂还可产生 400、480 和 $556 \mathrm{~nm}$ 等多个发光带 ${ }^{[15]}$, 而且这些发光的时间常数在 0.6 3 $\mu \mathrm{s}$ 之间变化, 属于慢衰减, 因此有人把纯 CsI 晶体中的慢衰减成分归因于杂质发光。但本实验显 示，即便是用同一批原料所生长的 CsI 晶体，其慢 分量的强度也会存在很大的差异, 因此难以用原料 中的固有杂质来解释慢分量的产生。相反, 同一原 料经不同的工艺生长所得晶体的快/慢分量比也差 异很大，因此其成因还有待进一步深究。

\section{3 时间特性}

\subsection{1 脉冲 $X$ 射线激发下的衰减时间}

以图 3 中的 8\#纯 CsI 样品为研究对象, 采用 GD40 光电管(响应时间为 $1 \mathrm{~ns}$ )测量该晶体在脉冲 X 射线激发下输出的光信号，同时采用另一只 GD40 配合 ST401 晶体监测 X 射线源的信号变化。整个测 
量系统(含 GD40 光电管、信号传输电缆和 DPO4104)

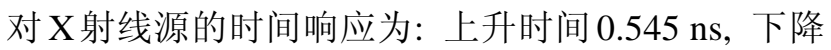
时间 $1.248 \mathrm{~ns}$, 半宽 $0.889 \mathrm{~ns}$ 。

闪鞦体的发光包括两个过程: 发光增加过程和 发光衰减过程, 前者包括入射粒子在晶体中的能量 丢失、电离、激发和发射光子数达到最大值的过程, 这个过程所需要的时间被称为上升时间; 后者则是 指发光强度从最大值衰减到最大值的 $1 / \mathrm{e}$ 所经历的 过程, 这个过程所需要的时间被称为发光衰减时 间。图 4 为 8\#纯 CsI 晶体对脉冲 X 射线的响应波形, 详细数据参见表 1 。通过对多次实验中 “GD40+ ST401” 的监测波形进行比较, 可以确认脉冲 X 射 线源状态比较稳定, 从而拟合出 8\#纯 CsI 晶体的平

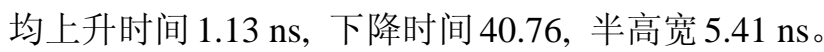

根据文献[10-12]中 CsI 有两种发光成分的描述, 对 CsI 晶体实测波形的下降沿波形采用二阶指数拟 合, 即

$$
I(t)=I_{1} / \tau_{1} \cdot \exp \left(-t / \tau_{1}\right)+I_{2} / \tau_{2} \cdot \exp \left(-t / \tau_{2}\right)
$$

拟合得到的衰减时间常数分别为 $\tau_{1}=3 \mathrm{~ns}$ 和 $\tau_{2}=22 \mathrm{~ns}$ 。 该值与 Schotanus 用 ${ }^{137} \mathrm{Cs}$ 和 $5.15 \mathrm{MeV} \alpha$ 粒子激发所 测得的衰减时间(2.1 ns 和 $21.7 \mathrm{~ns})$ 几乎相同 ${ }^{[9]}$, 但与 Woody 报道的 $\tau_{1}=7 \mathrm{~ns}$ 和 $\tau_{2}=29 \mathrm{~ns}^{[13]}$, Amsler 的 $\tau_{1}=6$ $\mathrm{ns}$ 和 $\tau_{2}=29 \mathrm{~ns}^{[12]}$ 和 Kubota 的 $\tau_{1}=10 \mathrm{~ns}$ 和 $\tau_{2}=36 \mathrm{~ns}^{[6]}$ 相比, 本文拟合出的衰减时间要略快一些。图 5 中 原始波形下降沿中的振荡是由于脉冲 $\mathrm{X}$ 射线源产生 的电磁振荡带来的, 振荡对下降沿波形拟合会带来 一定的不利影响。

\subsubsection{CsI 晶体对宇宙射线的响应时间}

根据符合法原理, 利用两支 ETL9815B 光电倍 增管(简称 PMT)同时测量同一块 CsI(pure)晶体对宇 宙射线的响应信号。两支 PMT 后端的信号传输电 缆长度相同, 分别接入 DPO4104 示波器的两个独立 信道。当两支 PMT 同时测量到脉冲波形信号时, 即 认为该波形为 CsI(pure) 在宇宙射线激励下发出的光 信号。

图 6 为 CsI 晶体对宇宙射线响应的一组符合波

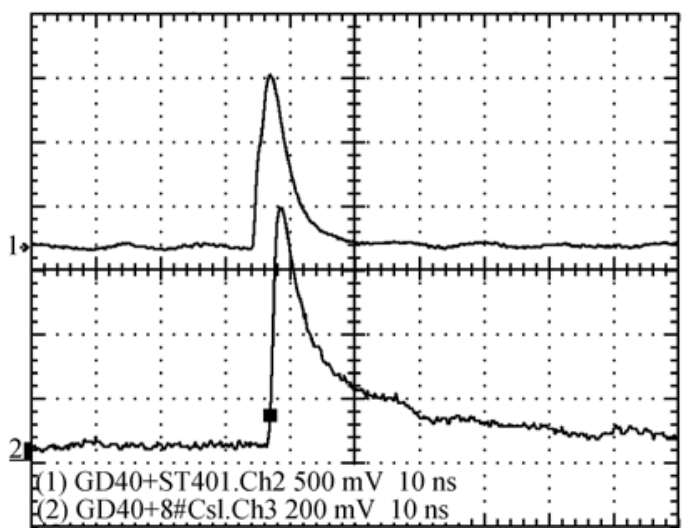

图 4 8\#CsI 晶体对脉冲 X 射线的响应波形

Fig. 4 Response of pure CsI crystal to the pulse X-ray

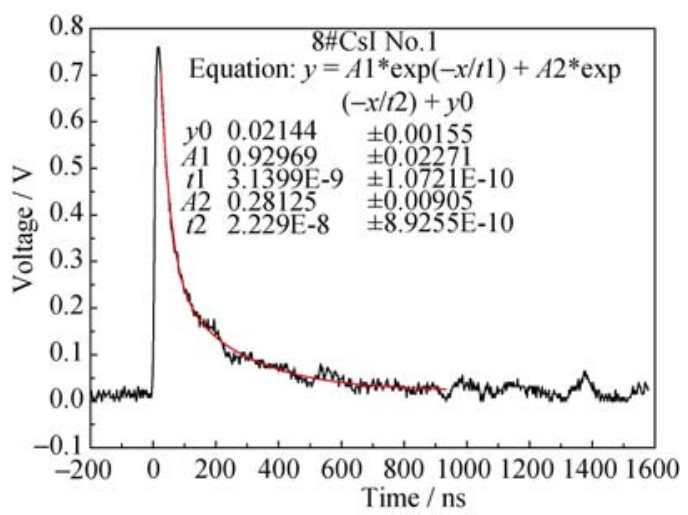

图 5 脉冲 X 射线激发下 8\#CsI 晶体的下降沿波形拟合

Fig. 5 Decay curve and its fitted line of 8\# CsI crystal excited by pulse X-ray

形，图中信道 1(Ch1)和信道 2(Ch2)为两支 PMT 同时 测量得到的波形。两个信号峰值时刻的时间差是由 于两路测量系统(PMT、信号电缆和示波器信道)的 时间响应差别造成的。图 6 中两支 PMT 测量得到的 波形信号形状基本一致。对多组波形信号的时间参 数取平均值得到的结果列于表 2 中。

表 2 中 CsI 晶体的上升时间响应较表 1 中所列 数据要慢一些, 这主要是由于表 2 实验中使用的 PMT 自身时间响应长达 $3 \mathrm{~ns}$ 左右, 比表 1 实验中使用的 光电倍增管时间响应要慢。考虑到两次实验中使

表 18 \#CsI 对脉冲 X 射线的时间响应参数

Table 1 Response of 8 \# CsI crystal to the pulse X-ray

\begin{tabular}{|c|c|c|c|c|c|c|}
\hline \multirow{2}{*}{ Test No. } & \multicolumn{3}{|c|}{ 8\#CsI } & \multicolumn{3}{|c|}{ Reference } \\
\hline & Rise time/ns & Decaytime/ns & FWHM/ns & Rise time/ns & Decaytime/ns & FWHM/ns \\
\hline 1 & 1.07 & 37.31 & 5.29 & 1.85 & 7.96 & 4.79 \\
\hline 2 & 1.16 & 39.88 & 5.36 & 1.86 & 7.85 & 4.97 \\
\hline 3 & 1.17 & 44.96 & 5.50 & 1.95 & 8.40 & 4.90 \\
\hline 4 & 1.10 & 40.88 & 5.49 & 1.85 & 7.25 & 4.80 \\
\hline mean & 1.13 & 40.76 & 5.41 & 1.88 & 7.87 & 4.87 \\
\hline
\end{tabular}


表 2 CsI(pure)晶体对宇宙射线的时间响应

Table 2 Response of 8\# pure CsI crystal to the cosmic ray

\begin{tabular}{cccc}
\hline Sample & Rise time $/$ ns & Decay time/ns & FWHM/ns \\
\hline 8\#CsI(pure) & 3.678 & 25.509 & 8.003 \\
\hline
\end{tabular}

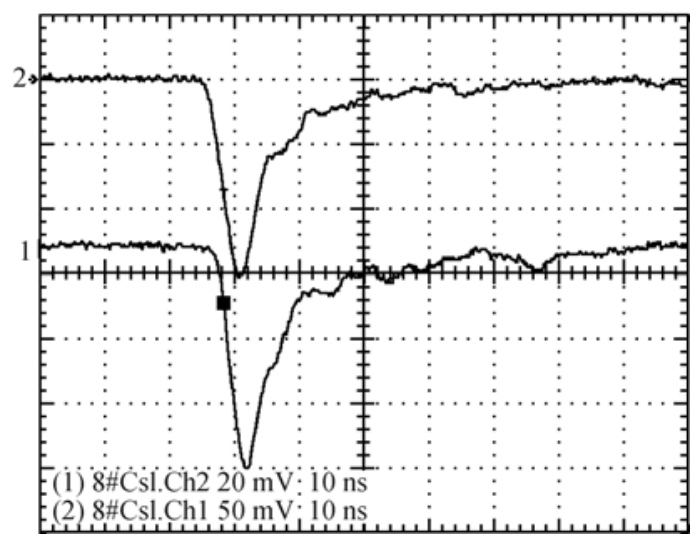

图 6 8\#CsI 晶体对宇宙射线的响应波形

Fig. 6 Response of 8\# CsI crystal to the cosmic ray

用的光电转换器件时间响应上的差别, 可以认为两 次实验的结果基本一致。脉冲 X 射线响应实验中光 电倍增管的时间响应更快, 对测试波形时间性能的 贡献更小, 因此认为该测试结果更加接近晶体自身 的性能。

两次实验中，信号波形的下降沿都有一个明显的 平台, 这是晶体发光中慢成分发光与快成分发光叠加 在一起造成的。根据图 7 中对实验波形下降沿的二阶
指数拟合结果，两个成分的发光衰减时间分别为 $\tau_{1}=2 \sim 3 \mathrm{~ns}$ 和 $\tau_{2}=18 \sim 25 \mathrm{~ns}$ 。这个结果与脉冲 $\mathrm{X}$ 射线响 应实验的结果完全吻合, 与大多数文献报道的数据 相比(表 3), $\tau_{2}$ 基本吻合，只有 $\tau_{1}$ 差异较大。对于衰减 时间在 ns 级的信号, 无论是探测系统还是数据传输/ 采集/记录系统, 或是数据的处理, 都存在较大的不 确定度, 各种系统因素和环境因素的影响也会在测 试过程中有所体现。另外，根据所测晶体紫外吸收边 更接近其理论吸收边的事实, 我们有理由认为高的 晶体质量也是其光衰减速度加快的原因之一。

\section{4 光输出与快慢分量比}

以 ${ }^{22} \mathrm{Na}$ 为激发源和 HAMAMATSU 的 R2059 光 电倍增管(PMT)为光探测器, 用 Tyvek 包裹尺寸为 $30 \mathrm{~mm} \times 30 \mathrm{~mm} \times 200 \mathrm{~mm}$ 的纯 CsI 晶体。为了比较 晶体籽晶端 $(\mathrm{A})$ 和尾端 $(\mathrm{B})$ 两端的差异, 分别以 $\mathrm{A}($ 籽 晶端 $) 、 B($ 尾端 $)$ 端与 PMT 直接耦合, 测得它们在不 同时间门宽下的多道能谱, 拟合出的光电子产额随 时间门宽的变化如图 8 所示。由图 8 可见, 两个端 面耦合所测得的衰减时间几乎相同，分别是 $29 \mathrm{~ns}$ 和 $28 \mathrm{~ns}$, 对应的光输出：籽晶端(A)为 143 p.e/MeV, 尾端 127 p.e./MeV，两者相差 $12.6 \%$ 。显然，籽晶端
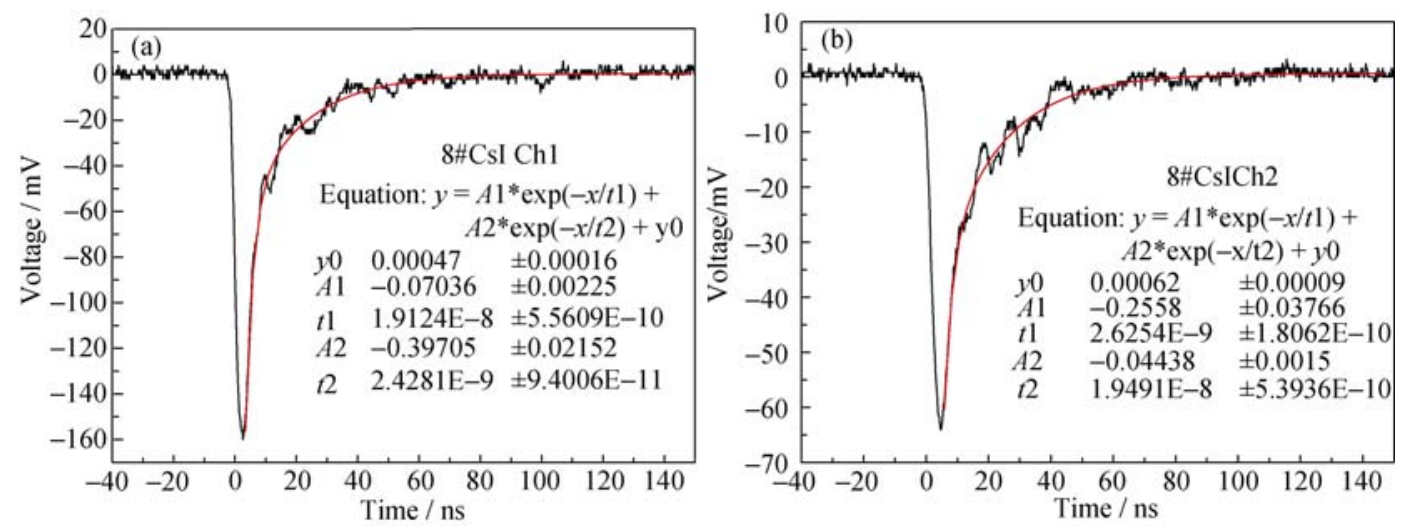

图 7 宇宙射线激发下从信道 1(a) 和信道 2(b) 所测得的 CsI 晶体下降沿的波形及其拟合曲线

Fig. 7 Decay curves and their fitted lines from channel 1 and channel 2 of pure CsI crystal excited with cosmic ray

表 3 本文所测试的纯 CsI 衰减时间与以往文献报道值的对比

Table 3 Comparison of decay times of pure CsI reported previously and those tested in this work

\begin{tabular}{ccccccc}
\hline & Kubota & Woody & Schotanus & Amsler & Belsky & This work \\
\hline$\tau_{1} /$ ns & 10 & 7 & 2 & $6 \pm 1$ & $5-9$ & $2-3$ \\
$\tau_{2} /$ ns & 36 & 29 & 22 & $28 \pm 2$ & $10-25$ & $18-25$ \\
Reference & {$[6]$} & {$[13]$} & {$[9]$} & {$[12]$} & {$[16]$} & \\
\hline
\end{tabular}




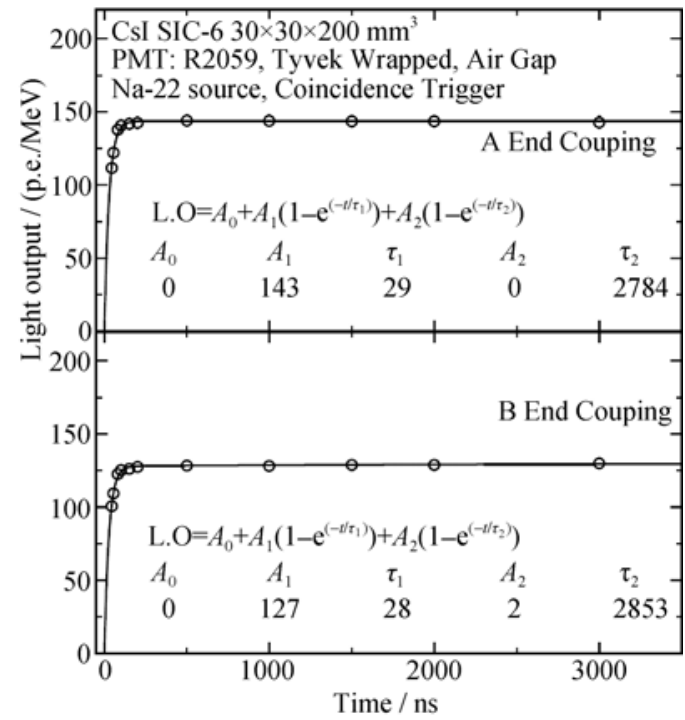

图 8 CsI 晶体分别用 A 端(上)、B 两端(下)耦合时在不同时 间门宽下所测得的光输出

Fig. 8 Light outputs of CsI crystal when it is coupled by A (top) and $\mathrm{B}$ (bottom) ends and integrated from different time gate

的晶体质量要优于尾端, 说明对于长尺寸晶体而言, 即便是本征发光, 依然存在发光不均匀性的现象。 值得注意的是, 从 $A 、 B$ 两端所测的慢分量分别为 0 和 2 p.e. $/ \mathrm{MeV}$, 超过 $100 \mathrm{~ns}$ 之后光输出不在随积分 时间的增加而变化, 呈现出一条水平直线, 说明与 慢分量有关的晶体缺陷在本次测试的样品中已经得 到有效抑制。

\section{3 结论}

纯碘化铯晶体的紫外截止吸收边约为 $240 \mathrm{~nm}$, 在可见光区的透过率达到 $80 \%$, 该值与理论计算值 非常接近。在紫外光和连续 X 射线激发下的发射波 长分别位于 $318 \mathrm{~nm}$ 和 $312 \mathrm{~nm}$ 附近, 除此之外, 它 们在 $440 \mathrm{~nm}$ 附近存在一个很宽的发光带, 前者属于 纯碘化铯晶体的本征发光, 后者则是与晶体缺陷有 关的发光。由于实验所测晶体的紫外截止吸收边最 接近理论值, 且发光成分中的慢分量低于已有的文 献报道值, 因此可认为实验生长的样品质量是迄今 为止最好的。同时, 无论以脉冲 X 射线或是宇宙射 线为激发源所测得纯 CSI 晶体的两个发光衰减时间 均为 2 3 ns 和 18 25 ns, 具有很好的一致性, 因此 提出本实验所测得纯碘化铯晶体的发光波长和衰 减时间更能代表该晶体的真实性能。这些结果进一 步确认了纯碘化铯晶体是一种难得的快衰减闪炼
材料。

致谢: 本文中的光输出数据是由美国加州理工学院 朱人元教授帮助测试的，作者在此特致谢意!

\section{参考文献:}

[1] RODNYI P A. Progress in fast scintillators. Radiation Measurements, 2001, 33: 605-614.

[2] BIASINIA, CASSIDY D B, DENG S H M, et al. Suppression of the slow component of scintillation light in $\mathrm{BaF}_{2}$. Nucl. Instrum. Methods. Phys. Res. A, 2005, 553: 550-558.

[3] GU MU, GAO PAN, LIU XIAO-LIN, et al. Crystal growth and characterization of CuI single crystals by solvent evaporation technique. Mater. Res. Bull., 2010, 45: 636-639.

[4] KUZMIN A. Endcap calorimeter for SuperBelle based on pure CsI crystals. Nucl. Instrum. Methods. Phys. Res. A, 2010, 623: 252-254.

[5] ATOANOV N, BARANOV V, BUDAGOV J, et al. Design and status of the Mu2e electromagnetic calorimeter. Nucl. Instrum. Methods. Phys. Res. A, 2016, 824: 695-698.

[6] KUBOTA S, SAKURAGI S, HASHIMOTO S, et al. A new scintillation material: Pure CsI with 10 ns decay time. Nucl. Instrum. Methods. Phys. Res. A, 1988, 268: 275-277.

[7] WEI ZONG-YING, ZHU REN-YUAN. Study on undoped CsI crystals. Nucl. Instrum. Methods. Phys. Res. A, 1993, 326: 508-512.

[8] WU Z, YANG B, TOWNSEND P D. Radioluminescence and thermoluminescence properties of X-ray-irradiated pure CsI. $J$. Luminescence, 2008, 128: 1191-1196.

[9] SCHOTANUS P, KAMERMANA R, DORENBOS P, et al. Scintillation characteristics of pure and Tl-doped CsI crystals. IEEE. Trans. Nuc.l Sci., 1990, 37(2): 177-182.

[10] SHEN DING-ZHONG, DENG QUN, YUAN XIANG-LONG, et al. Luminescent Performance of pure CsI crystals. Journal of Inorganic Materials, 1997, 12(3): 273-278.

[11] BABIN V, KRASNIKOV A, WIECZOREK H, et al. Luminescence of complicated thallium centers in CsI: Tl. Nucl. Instrum. Methods. Phys. Res. A, 2002, 486: 486-489.

[12] AMSLER C, GROGLER D, JOFFRAIN W, et al. Temperature dependence of pure CsI: scintillation light yield and decay time. Nucl. Instrum. Methods. Phys. Res. A, 2002, 480: 494-500.

[13] WOODY C L, LEVY P W, KIERSTEAD J A, et al. Readout techniques and radiation damage of undoped Cesium Iodide. IEEE. Trans. Nucl. Sci., 1990, 37(2): 492-499.

[14] HAMADA M M, NUNOY Y, KUBOTA S, et al. Suppression of the slow emission component in pure CsI by heat treatment. Nucl. Instrum. Methods. Phys. Res. A, 1995, 365: 98-103.

[15] BABIN V, KRASNIKOV A, NIKL M, et al. Luminescence and relaxed excited state origin in CsI: Pb crystals. J. Luminescence, 2003, 101: 219-226.

[16] BELSKY A N, VASILEV A N, MIKHAILIN V V, et al. Time-resolved XEOL spectroscopy of new scintillators based on CsI. Rev. Sci. Instrum., 1992, 63(1): 806-809. 